\title{
Effects of dance movement therapy on Chinese cancer patients: A pilot study in Hong Kong
}

\author{
Rainbow T. H. Ho Ph.D.*
}

Centre on Behavioral Health, Faculty of Social Sciences, The University of Hong Kong,

G/F, Pauline Chan Building, 10 Sassoon Road, Pokfulam, Hong Kong

\section{Introduction}

Dance movement therapy (DMT) is predicated on the belief that body movement reflects and affects psychological states. DMT has been widely used to treat people with mental and psychological problems (Goodill, 1987; Milliken, 2002; Romero, Hurwitz, \& Carranza, 1983; Stanton Jones, 1992), and has also been used during the past decade to reduce the special stress and anxiety associated with chronic diseases (Goodill, 2005; Stewart, McMullen, \& Rubin, 1994) and cancer (Brandberg, Manssonbrahme, Ringborg, \& Sjoden, 1995; Cohen \& Walco, 1999; Dibbell-Hope, 2000).

For most cancer patients, the treatment procedures are traumatic. The surgical removal of body parts, nausea, loss of hair, and fatigue from radiotherapy and chemotherapy internally assault the physical body, causing suffering and pain. Psychological and physical problems often appear after the medical treatment. Dance movement therapy, which acts directly on the body, may then have a profound effect on the healing process in cancer patients. Previous studies have indicated that physical exercises and dance movement could enhance the range of movement and freedom of total body movement, create a positive mood, and facilitate the psychological adjustment to a diagnosis of cancer, thereby improving quality of life in breast cancer patients (Courneya, Keats, \& Turner, 2000; Mock et al., 1997; Molinaro, Kleinfeld, \& Lebed, 1984, 1986; Rowden, 1984; Sandel \& Judge, 2004).

\footnotetext{
* Tel.: +852 2589 0506; fax: +852 28166710 .

E-mail address: tinho@hkucc.hku.hk.
} 
Where a more in-depth approach such as authentic movement has been used, dance movement therapy has helped significantly to increase participants' energy and reduce fatigue. Objective and self-perceived subjective improvements have also been found to patients' mood, body image, and self-esteem (Dibbell-Hope, 2000). Participants indicated that they have increased sense of hope, ease, strength and social support, while negative mood and worry about future was decreased. Serlin, Classen, Frances, and Angell (2000) also revealed that breast cancer patients who took part in a dance movement therapy group became less depressed and anxious, and significant improvements were observed in their fatigue, anxiety, and tension scales.

Evidence also indicates that taking part in dance movement therapy groups has helped to reduce stress in both young and adult cancer patients (Bojner-Horwitz, Theorell, \& Maria Anderberg, 2003; Cohen \& Walco, 1999). Since stress plays an important role in cancer development and prognosis (Abercrombie et al., 2004; Lillberg et al., 2003; Luecken \& Compas, 2002; Lundstrom \& Furst, 2003; Ramirez et al., 1989; Sephton \& Spiegel, 2003), the effect of DMT on stress and stress-related physiology has attracted considerable attention recently. Although little research has yet been conducted into the effect of DMT on stress physiology, one study has indicated that DMT has a beneficial effect on stress-related hormones (Bojner-Horwitz et al., 2003).

However, knowing that Chinese people are not used to moving in front of others due to strong impulse control and self-discipline (Ho, 1986; $\mathrm{Ho}, \mathrm{Fu}, \& \mathrm{Ng}$, 2004), techniques used in traditional DMT approaches which emphasize spontaneous movement and improvisation may not be appropriate for Chinese people, at least not at the beginning of the program. On the other hand, Chinese people usually have greater respect for authority, and stronger preference for practical and immediate solutions to problems than people in the West (Leung \& Lee, 1996; Sue \& Sue, 1990). Hence, an educational approach may be more appropriate. Treatment may start with teaching dances and steps, and slowly transit to self-initiated movement. Since a similar approach had been used in teaching creative dance classes by the author and was successful, it may be interesting to know if this approach can also be applied to Chinese cancer patients in a DMT group.

In view of the holistic healing effect of DMT, its unique advantage in addressing psychological problems at the bodily level, and its applicability to people who have been diagnosed with cancer, a pilot DMT group was organized for Chinese cancer patients in Hong Kong. This was the first formal DMT program run in Hong Kong for cancer patients. Its primary aim was to help cancer patients regain a sense of comfort and take pleasure from their bodies through dancing and relaxation exercises. Its secondary aim was to help them to use their own bodies to express their feelings more openly. The suitability of the treatment approach that is different from traditional DMT approaches was also evaluated. Since relaxation, dancing and exercise may help to reduce stress, a Perceived Stress Scale (PSS; Cohen, 1988) was used to measure the change in stress level before and after the DMT sessions. Furthermore, since an overall improvement in levels of distress, vigor and self-expression may also lead to an improvement in patients' self-image and self-esteem, the Rosenberg Self-Esteem Scale (SES; Rosenberg, 1965) was used to understand these changes. It was hypothesized that both stress and self-esteem would be improved after the DMT program. 


\section{Methods}

\section{Study design}

This pilot study used a pre- and post-study design. Participants were asked to complete questionnaires at the beginning of the first session and also after the end of the last session. Besides providing psychometric data, they were also required to complete an evaluation form that explored how they felt about the DMT program.

\section{Participants}

Participants were recruited from the Cancerlink Support and Resource Centre. Program pamphlets were displayed in the center. Participants who were interested in the program registered with the center workers. Informed consent was obtained from participants before the program started.

\section{DMT program}

A 90-min DMT session was held once a week for six consecutive weeks. Each session had a specific theme and the process included warm-up exercises, dance, thematic movement, and relaxation exercises. Sessions usually started with a general body warm-up, followed by a specific dance. A different dance was taught in each session and participants enjoyed both learning new dances and repeating old ones. Steps, modified by the author, were very simple and a variety of dance types were tried, including waltzes, Chinese folk dances, country line dances, Western character dances, Middle Eastern dances, and social dances. Interaction between group members was encouraged through movement games. Thematic movement was developed according to the progress and dynamics of the group. The themes included body expression, opening and closing, dance of love, dancing from the heart, and dancing from the soul. Sessions usually ended with relaxation exercises and guided imagery. Simple stretching exercises and selected movement from yoga were taught, and guided imagery was conducted to help participants to relax and cool down.

\section{Measurements}

In order to measure the effect of the DMT program on stress and self-esteem in participants, Perceived Stress and Rosenberg Self-Esteem Scales were used before and after intervention. Participants were also asked to complete the program evaluation form at the end of the last session.

\section{Perceived Stress Scale (PSS)}

The Perceived Stress Scale used in this study was a 10-item scale (Cohen, 1988). It was adapted from the original 14-item scale to measure the degree to which a situation in an individual's life is perceived as stressful (Cohen, Kamarck, \& Mermelstein, 1983). PSS has been widely used to measure the stress level in Chinese indicating its good reliability and validity (Chen, 1994; Ho, Chan, \& Ho, 2004; Lai, 1996; Lee \& Crockett, 1994). A higher 
score indicates a higher perceived stress level. Cronbach's coefficient alpha was 0.7 and 0.9 in pre- and post-test analyses, respectively, in the present study.

\section{Rosenberg Self-Esteem Scale (SES)}

The Rosenberg Self-Esteem Scale was a 10-item measure for evaluation of self-esteem, revealing individuals' positive or negative orientation towards themselves (Rosenberg, 1965). The Chinese version of this scale was validated and had been used in some studies on Chinese subjects indicating its acceptable validity and reliability (Berk, Cooper, Liu, \& Marazita, 2001; Farruggia, Chen, Greenberger, Dmitrieva, \& Macek, 2004; Lee \& Lee, 2000). A higher score indicates higher self-esteem. Cronbach's coefficient alpha was 0.6 and 0.7 in pre- and post-test analyses, respectively, in this study.

\section{Evaluation form}

Participants were asked to complete a written evaluation form at the end of the last session to indicate levels of satisfaction with the program and how they felt about it.

\section{Data analysis}

Means and standard deviations were calculated for each measure. Although the sample size was small $(N<30)$, the distribution of data did not vary too much from a normal distribution. A $t$-test was thus conducted to see if there was any significant change before and after the DMT program. The effect size, which is independent of the sample size, was also calculated for each measure, to give some impression of the program's effectiveness.

\section{Results}

\section{Participants}

Twenty-two cancer patients aged from 39 to 69 were recruited. The average age was 50.18 (S.D. $=7.08)$. Two $(9.1 \%)$ participants had completed primary education; $15(68.2 \%)$ had secondary education; $2(9.1 \%)$ had matriculated; and $3(13.6 \%)$ were university graduates. Most participants were married $(n=19,86.4 \%)$; only three were single $(13.6 \%)$. The cancer types included breast cancer $(n=15,68.2 \%)$, colon cancer $(n=1,4.5 \%)$, leukemia $(n=1$, $4.5 \%)$, lymphoma $(n=2,9.1 \%)$, and nasopharyngeal carcinoma $(n=3,13.6 \%)$.

Table 1

Changes of PSS and SES before and after DMT

\begin{tabular}{lllrl}
\hline & Pre, mean (S.D.) & Post, mean (S.D.) & $t(p)$ & Effect size $(d)$ \\
\hline Perceived Stress Scale & $23.31(5.61)$ & $20.38(6.75)$ & $2.22(0.042)$ & 0.49 \\
Self-Esteem Scale & $16.67(3.29)$ & $18.20(3.65)$ & $-1.77(0.099)$ & 0.46 \\
\hline
\end{tabular}

Pre: Before the first DMT session.

Post: At the end of the sixth DMT session. 
Perceived Stress Scale (PSS) and Self-Esteem Scale (SES) Scores Before (Pre) and After (Post) DMT

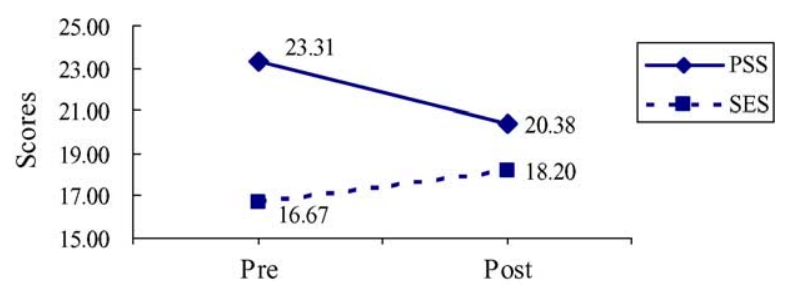

Fig. 1. Changes of PSS and SES before and after DMT.

Of the 22 participants, 16 of them provided both pre-and post-test data. Data analysis was thus conducted for these 16 responses. Three participants did not come to the last session due to personal reasons (going for follow-up body check and family trip), thus only 19 participants completed the program evaluation.

\section{Stress and self-esteem}

Means and standard derivations of the measures are shown in Table 1. Scores on the Perceived Stress Scale were significantly lower after the DMT program $(t=2.22, p=0.042)$

Table 2

Participants' written responses to the DMT program

\begin{tabular}{ll}
\hline Themes & Participants' written responses \\
\hline Relaxation: & "It makes me feel less stressed." \\
Feeling relaxed, less stressed & "I feel relaxed and happy, I know more friends." \\
and happy & "Happy!" \\
& "My body is relaxed and fuses into the steps." \\
& "I am more relaxed and happier; my body gets a lot of stretching too." \\
& "I can be relaxed and less depressed." \\
"My body is relaxed and has become less tense." & "I can relax and release myself, do whatever I want to do." \\
Connection from body to mind, & "It is a release for me." \\
feeling released, and more & "My body and mind are both joyous." \\
open & "I can open up and become less self-centered." \\
Personal growth: & "I know more about myself." \\
Self-understanding, getting & "My mind is more clear and there are fewer intrusive thoughts." \\
control on negative thoughts & "I know how to manage my emotions; unlike before, I hide everything in \\
and emotions, less shy & my heart; previously I was too shy to perform in front of other people, \\
Spirituality: & now I am very happy to do that." \\
Connection from body, mind to & "Dancing can bring health to the body and comfort to the soul." \\
spirit, getting in touch with & "I can release myself and get in touch with my inner child." \\
inner child &
\end{tabular}


and with a medium effect size $(d=0.49)$. Self-esteem scores also improved, with a medium effect size of 0.46, but the change did not reach a statistically significant level $(t=-1.77$, $p=0.099$ ). Diagrammatic illustrations of the changes in the two scales are shown in Fig. 1.

\section{Program evaluation}

Nineteen participants responded to the evaluation. All respondents reported that the program helped them a lot in various aspects such as increasing their confidence to fight cancer, gaining support from others, and knowing more about rehabilitation. They believed that they could use what they learned from the program in their daily lives. Most of them felt relaxed, happy and less stressed. Some of them even gained beneficial effects on their mind and spirit. According to the content of written responses, four major themes were identified. They were: relaxation, mind-body interaction, personal growth, and spirituality (see Table 2).

\section{Discussion}

Although DMT is a completely new modality of psychotherapy for most Hong Kong people, the high participation rate and the positive feedback from participants indicated that the program was well-received by Chinese cancer patents. A significant reduction in perceived stress, with a medium effect size of 0.49 , and a positive change in self-esteem, with a medium effect size of 0.46 , also demonstrated the beneficial effect of the program. This confirmed the hypothesis. The insignificant improvement in self-esteem might be due to the small sample size and short duration of the monitoring period. Change in self-esteem might require more time to develop than the change in perceived stress. In this case, where both the therapy and follow-up period were brief, self-esteem may not be an appropriate dependent variable. Furthermore, the design of the program did not specifically address the problem of self-esteem, and hence its effect might not have been very significant. However, due to the small sample in this pilot study, the data should be interpreted with due caution. Further studies with a larger sample and with more specific therapeutic aims may provide more information on the effects of this kind of program on participants' self-esteem.

In their subjective evaluations, all participants viewed the program as helpful. They felt relaxed, comfortable, happy, and released, and started to open up and be less shy and tense. Some participants claimed to be able to get in touch with their inner world, and felt the connections between mind, body, and spirit. Participants' subjective feelings about the DMT strongly reflected the underlying working premise of DMT: the interconnectedness of mind and body. Dance and body movement may elicit changes in emotions and feelings. As Schmais observed, "... motor actions cause changes in psychic attitudes" (Schmais, 1985). Through working on the body, the mind can be changed and the soul can be enriched.

For cancer patients, the proposition that there was an interaction between mind and body was particularly important, as cancer traumatizes the body from the diagnostic stage to the invasive medical treatment stage. Most cancer patients experience a sense of loss of control over their own bodies, and (as nothing is certain with cancer) also a loss of direction in life. 
Through dance and body movement participants regained some sense of control over their bodies, and also learned to accept and enjoy whatever they had at that particular moment. They felt happier and less distressed, and had a better self-image. The group setting in which participants danced and played together, shared the rhythm of the music and talked together freely, also greatly reduced the sense of loneliness caused by cancer diagnosis. The group developed strong cohesion, and participants developed relationships with each other.

Another aim of the DMT program was to help participants to express themselves more by using their own bodies. At the beginning of the program some of the participants were quite reluctant to dance or move, although they quite enjoyed the relaxation exercises. They said that they did not know how to move and dance. This was a reasonable and expected response, as traditional Chinese culture emphasizes discipline and self-control and many Chinese people are educated to have a strong impulse to control both their physical movements and their emotional expression (Ho, 1986; Ho, Chan, et al., 2004; Ho, Fu, et al., 2004). In this program, to help participants to feel more confident in starting to dance, an incremental approach was adopted. Dance steps were taught first, and there was then a slow transition into dance improvisation. Feedback from participants indicated that this approach was welcomed. By the fifth session, all participants could dance spontaneously and on their own in front of others. Some of the participants also actively talked about their feelings and emotions associated with the movement and dance they were performing.

To conclude, this pilot DMT program for cancer patients achieved its aims of helping participants to take pleasure from their bodies and began to express their feelings and emotions more openly. The significantly lowered perceived stress scores and the improvement in self-esteem scores also indicate that the program was effective. A subjective evaluation of the program's effectiveness revealed that participants benefited at physical, psychological and spiritual levels. Although participants felt shy about dancing at the beginning of the program, the use of an incremental approach encouraged them to dance spontaneously in front of others by the fifth session. This reflected both their growing trust in the group relationship and their enjoyment in dancing. It should be noted that this was something of an achievement for Chinese cancer patients, as Chinese people are not accustomed to dancing or moving in front of others. Further improvement in kinesthetic expression may be possible using a longer and more focused program.

Since this is the first DMT program for cancer patients in Hong Kong, the program was relatively short and was of an explorative nature. The overall changes observed in this study might be due to the healing process of DMT and its simultaneous influence on body, mind, and spirit. However, the small sample and the lack of a comparison group limit the efficacy of this study. Care should be taken not to generalize too far from the results. A further study, based on a longer program with a more in-depth approach and using a larger sample with a control group, might provide more useful information on the impact of DMT on cancer patients and the influence, if any, of cultural factors on its use.

\section{Acknowledgments}

The author would like to thank the Hong Kong Cancer Fund and the Cancerlink Support and Resource Center for supporting the Dance Movement Therapy Program. She is 
particularly grateful for the help given by Ms Wong Kam Fung, the Center's manager, throughout the program. The author would also like to express her sincere gratitude to all the participants in this study, and to thank the University's technical writer, Dr David Wilmshurst, for commenting on an early draft of this paper.

\section{References}

Abercrombie, H. C., Giese-Davis, J., Sephton, S., Epel, E. S., Turner-Cobb, J. M., \& Spiegel, D. (2004). Flattened cortisol rhythms in metastatic breast cancer patients. Psychoneuroendocrinology, 29(8), 1082-1092.

Berk, N. W., Cooper, M. E., Liu, Y. E., \& Marazita, M. L. (2001). Social anxiety in Chinese adults with oral-facial clefts. Cleft Palate-Craniofacial Journal, 38(2), 126-133.

Bojner-Horwitz, E., Theorell, T., \& Maria Anderberg, U. (2003). Dance/movement therapy and changes in stressrelated hormones: A study of fibromyalgia patients with video-interpretation. The Arts in Psychotherapy, 30(5), 255-264.

Brandberg, Y., Manssonbrahme, E., Ringborg, U., \& Sjoden, P. O. (1995). Psychological reactions in patients with malignant-melanoma. European Journal of Cancer, 31A(2), 157-162.

Chen, C. H. (1994). An exploration of postpartum depression model using LISREL. Gaoxiong Yi Xue Ke Xue Za Zhi, 10(5), 229-238.

Cohen, S. (1988). Perceived stress in a probability sample of the United States. In S. Spacapan \& S. Oskamp (Eds.), The social psychology of health. The Claremont Symposium on Applied Social Psychology (pp. 31-67). Thousand Oaks, CA: Sage Publications, Inc.

Cohen, S., Kamarck, T., \& Mermelstein, R. (1983). A global measure of perceived stress. Journal of Health and Social Behavior, 24(4), 385-396.

Cohen, S. O., \& Walco, G. A. (1999). Dance/movement therapy for children and adolescents with cancer. Cancer Practice, 7(1), 34-42.

Courneya, K. S., Keats, M. R., \& Turner, A. R. (2000). Physical exercise and quality of life in cancer patients following high dose chemotherapy and autologous bone marrow transplantation. Psycho-Oncology, 9(2), 127-136.

Dibbell-Hope, S. (2000). The use of dance/movement therapy in psychological adaptation to breast cancer. The Arts in Psychotherapy, 27(1), 51-68.

Farruggia, S. P., Chen, C. S., Greenberger, E., Dmitrieva, J., \& Macek, P. (2004). Adolescent self-esteem in crosscultural perspective-Testing measurement equivalence and a mediation model. Journal of Cross-Cultural Psychology, 35(6), 719-733.

Goodill, S. W. (1987). Dance/movement therapy with abused children. The Arts in Psychotherapy, 14(1), 59-68.

Goodill, S. W. (2005). Dance/movement therapy for adults with cystic fibrosis: Pilot data on mood and adherence. Alternative Therapies in Health and Medicine, 11(1), 76-77.

Ho, D. Y. F. (1986). Chinese patterns of socialization: A critical review. In M. H. Bond (Ed.), The psychology of the Chinese people (pp. 1-37). Hong Kong: Oxford University Press.

Ho, R. T. H., Chan, C. L. W., \& Ho, S. M. Y. (2004). Emotional control in Chinese female cancer survivors. Psycho-Oncology, 13(11), 808-817.

Ho, D. Y. F., Fu, W., \& Ng, S. M. (2004). Guilt, shame and embarrassment: Revelations of face and self. Culture \& Psychology, 10(1), 64-84.

Lai, J. C. L. (1996). Perceived stress and physical symptoms: The problem of the response set of social desirability in Hong Kong undergraduates. Psychologia, 39(1), 50-54.

Lee, S., \& Crockett, M. S. (1994). Effect of assertiveness training on levels of stress and assertiveness experienced by nurses in Taiwan, Republic of China. Issues In Mental Health Nursing, 15(4), 419-432.

Lee, S., \& Lee, A. M. (2000). Disordered eating in three communities of China: A comparative study of female high school students in Hong Kong, Shenzhen, and rural Hunan. International Journal of Eating Disorders, 27(3), 317-327.

Leung, P.W.L., \& Lee, P.W.H. (1996). Psychotherapy with the Chinese. In M.H. Bond (Ed.), The handbook of Chinese psychology (pp. 441-456). Hong Kong: Oxford University. 
Lillberg, K., Verkasalo, P. K., Kaprio, J., Teppo, L., Helenius, H., \& Koskenvuo, M. (2003). Stressful life events and risk of breast cancer in 10,808 women: A cohort study. American Journal of Epidemiology, 157(5), 415-423.

Luecken, L. J., \& Compas, B. E. (2002). Stress, coping, and immune function in breast cancer. Annals of Behavioral Medicine, 24(4), 336-344.

Lundstrom, S., \& Furst, C. J. (2003). Symptoms in advanced cancer: Relationship to endogenous cortisol levels. Palliative Medicine, 17(6), 503-508.

Milliken, R. (2002). Dance/movement therapy as a creative arts therapy approach in prison to the treatment of violence. The Arts in Psychotherapy, 29(4), 203-206.

Mock, V., Dow, K. H., Meares, C. J., Grimm, P. M., Dienemann, J. A., Haisfield-Wolfe, M. E., et al. (1997). Effects of exercise on fatigue, physical functioning, and emotional distress during radiation therapy for breast cancer. Oncology Nursing Forum, 24(6), 991-1000.

Molinaro, J., Kleinfeld, M., \& Lebed, S. (1984). Dance-A therapeutic modality for the breast surgery patient. Physical Therapy, 64(5), 749-749.

Molinaro, J., Kleinfeld, M., \& Lebed, S. (1986). Physical therapy and dance in the surgical-management of breast-cancer-A clinical report. Physical Therapy, 66(6), 967-969.

Ramirez, A. J., Craig, T. K., Watson, J. P., Fentiman, I. S., North, W. R., \& Rubens, R. D. (1989). Stress and relapse of breast cancer. British Medical Journal, 298(6669), 291-293.

Romero, E. F., Hurwitz, A. J., \& Carranza, V. (1983). Dance therapy on a therapeutic community for schizophrenic patients. The Arts in Psychotherapy, 10(2), 85-92.

Rosenberg, M. (1965). Society and the adolescent self-image. Princeton, New Jersey: Princeton University Press.

Rowden, R. (1984). Therapeutic pleasure-An unexpected setting for dance-As part of the routine in a cancer hospital. Dance and Dancers, 409, 32-33.

Sandel, S. L., \& Judge, J. O. (2004). Dance and movement program improves quality of life measure in breast cancer survivors. Paper presented at the 39th Annual American Dance Therapy Association Conference, New Orleans, Louisiana.

Schmais, C. (1985). Healing processes in group dance therapy. American Journal of Dance Therapy, 8, 17-36.

Sephton, S., \& Spiegel, D. (2003). Circadian disruption in cancer: A neuroendocrine-immune pathway from stress to disease? Brain Behavior and Immunity, 17(5), 321-328.

Serlin, I. A., Classen, C., Frances, B., \& Angell, K. (2000). Symposium: Support groups for women with breast cancer: Traditional and alternative expressive approaches. The Arts in Psychotherapy, 27(2), 123-138.

Stanton Jones, K. (1992). An introduction to dance movement therapy in psychiatry. New York, NY: Tavistock/Routledge.

Stewart, N. J., McMullen, L. M., \& Rubin, L. D. (1994). Movement therapy with depressed inpatients: A randomized multiple single case design. Archives of Psychiatric Nursing, 8(1), 22-29.

Sue, D. W., \& Sue, D. (1990). Counseling the culturally different (2nd ed.). New York: John Wiley \& Sons. 